\title{
Hybrid Ameloblastoma in the Anterior Mandible
}

\author{
Mandana Pourian', Mersad Mehranahad², Pooneh Dehghan² \\ ${ }^{1}$ Radiology Department, Rasool Akram Hospital, Iran University of Medical Sciences, Tehran, Iran \\ ${ }^{2}$ Taleghani Hospital Research Department, Shahid Beheshti University of Medical Sciences, Tehran, Iran \\ Email: mandanapourian@gmail.com
}

Received 29 June 2015; accepted 29 August 2015; published 1 September 2015

Copyright (c) 2015 by authors and Scientific Research Publishing Inc.

This work is licensed under the Creative Commons Attribution International License (CC BY).

http://creativecommons.org/licenses/by/4.0/

(c) (i) Open Access

\begin{abstract}
Ameloblastoma-a benign epithelial odontogenic tumor-histologically occurs in different patterns, the most common of which are plexiform and follicular. "Hybrid" ameloblastoma is a variant in which areas of follicular or plexiform pattern (generally called convention alameloblastoma) coexist with areas of pronounced stromal desmoplasia (desmoplastic ameloblastoma). They most often occur in the mandibular ramus. We present a case of "hybrid" lesion-desmoplastic and conventional ameloblastoma-in the anterior mandible, focusing on its' imaging features.
\end{abstract}

\section{Keywords}

Odontogenic Tumor, Hybrid, Conventional, Desmoplastic, Ameloblastoma

\section{Introduction}

Ameloblastoma is a benign—but locally aggressive-epithelialodontogenic tumor. The incidence of this tumor varies in different studies (1\% - 62\% of all odontogenic tumors) [1] [2]. Six different histopathological variants of ameloblastoma are mentioned: desmoplastic, granular cell, basal cell, plexiform, follicular and acanthomatous, the most common of which are plexiform and follicular (each comprising one third of the patients).

"Hybrid" ameloblastoma is a rare variant (about 1\% - 4\% of ameloblastomas) [3]-[5] in which typical areas of follicular or plexiform pattern (generally called onventional ameloblastoma) coexist with areas of pronounced stromaldesmoplasia (desmoplastic ameloblastoma). They most often (80\%) occur in the mandible. Although conventional ameloblastoma has no gender difference in prevalence [1], desmoplastic variant is seen more in men [5]; whereas hybrid ameloblastoma has slight female predilection with M:F ratio 11:16 [6], and mostly in the fourth and fifth decades of life (mean age of 36) [1] [7] [8]. The most prevalent symptom is facial swelling [1]; but as the tumor is locally aggressive, it can destroy surrounding tissues. 
Ameloblastoma is demonstrated as a radiolucent area which may present in three different forms: unicystic, multicystic and peripheral. The most common form (50\% of cases) is the multicystic type with cystic aggregates separated by bony septae (soap bubble pattern) [1]. Unicystic ameloblastomas occur in younger patients (mainly second to third decades). We present a case of "hybrid" (desmoplastic and conventional) ameloblastoma in the anterior mandible with emphasis on the imaging features.

\section{Clinical Summary}

A 39-year-old male presented to ear-nose-throat clinic complaining of painless expansion of his lower jaw for approximately one year. He was a teacher and had no past medical conditions. Clinical examination showed localized swelling in the left paramesial region of mandible. In the oral cavity, osseous expansion with normal appearing mucosal covering was evident. Dental malocclusion was present over the lesion: displaced alveolar processes, dental loss and loosening of the teeth.

On plain x-ray, an approximately 5 - $6 \mathrm{~cm}$ mixed lytic-sclerotic expansile lesion with ill-defined outline was shown in the mandibular body with involvement of alveolar processes (Figure 1). CT scans (bone window) of the lesion revealed a partially destructive, lyticexpansile lesion with thickening of the remaining bony trabeculae in the mandibular body. In soft tissue window, subcutaneous involvement of the left mandibular region was present (Figure 2).

Incisional biopsy put the pathological diagnosis of desmoplastic ameloblastoma. The lesion was then surgically resected (enucleation) with $1.5 \mathrm{~cm}$ safe-margin and prosthetic plate was substituted in thedefect. At this time, pathology survey of the samples brought forth the diagnosis of conventional ameloblastoma. Samples from separate points of the lesion showed different types of ameloblastoma: desmoplastic and conventional. Therefore, the diagnosis of "Hybrid" ameloblastoma was established.

\section{Histopathological Findings}

Microscopic sections showed histologic features of desmoplasticamel oblastoma in the medial portion, and those of follicularamel oblastoma with cystic change in the lateral portion of the lesion. Two regions were located distinctly: in the desmoplastic part, epithelial cell islands and cords were located in a densely collagenized connective stroma without peripheral palisading of columnar ameloblastic cells. In the follicular portion of the lesion, tumor nests were composed of peripheral palisading (columnar) differentiation and central stellate reticulum-like cells. These cells showed cystic degeneration and squamous metaplasia in some areas.

\section{Discussion}

Ameloblastoma is probably one of the most controversial neoplasms of the facial skeleton. It is believed that the earliest reference to this tumor was made in 1868 by Broca and was considered at that time to be of odontogenic origin [8].

Based on WHO definition, it is a locally invasive neoplasm which often has a follicular or plexiform pattern in a fibrous stroma. In the mandible (80\% of ameloblastomas), $70 \%$ are located in the area of molars or the ramus, $20 \%$ in the premolar region, and $10 \%$ in the anterior region [1]. It appears as a radiolucent lesion and presents in three radio graphicpatterns, the most common of which is the multi-locular with soap bubble pattern [9]. It is usually asymptomatic unless it becomes large or cause deformity and could lead to oral complications.

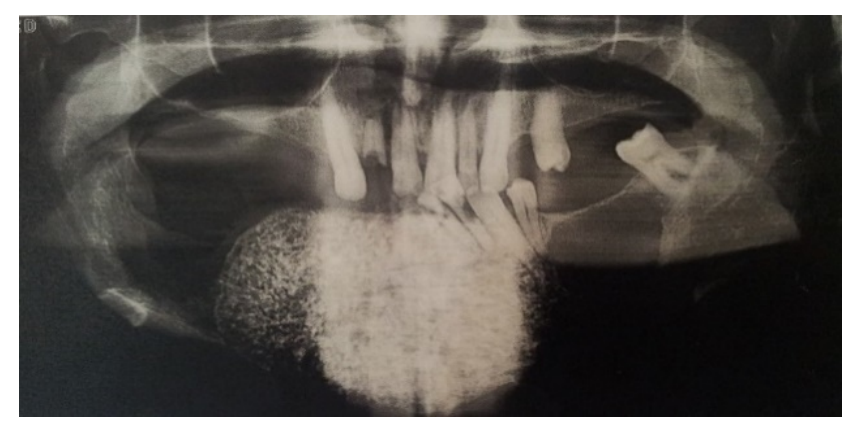

Figure 1. Panoramic X-ray radiography of the mouth. 

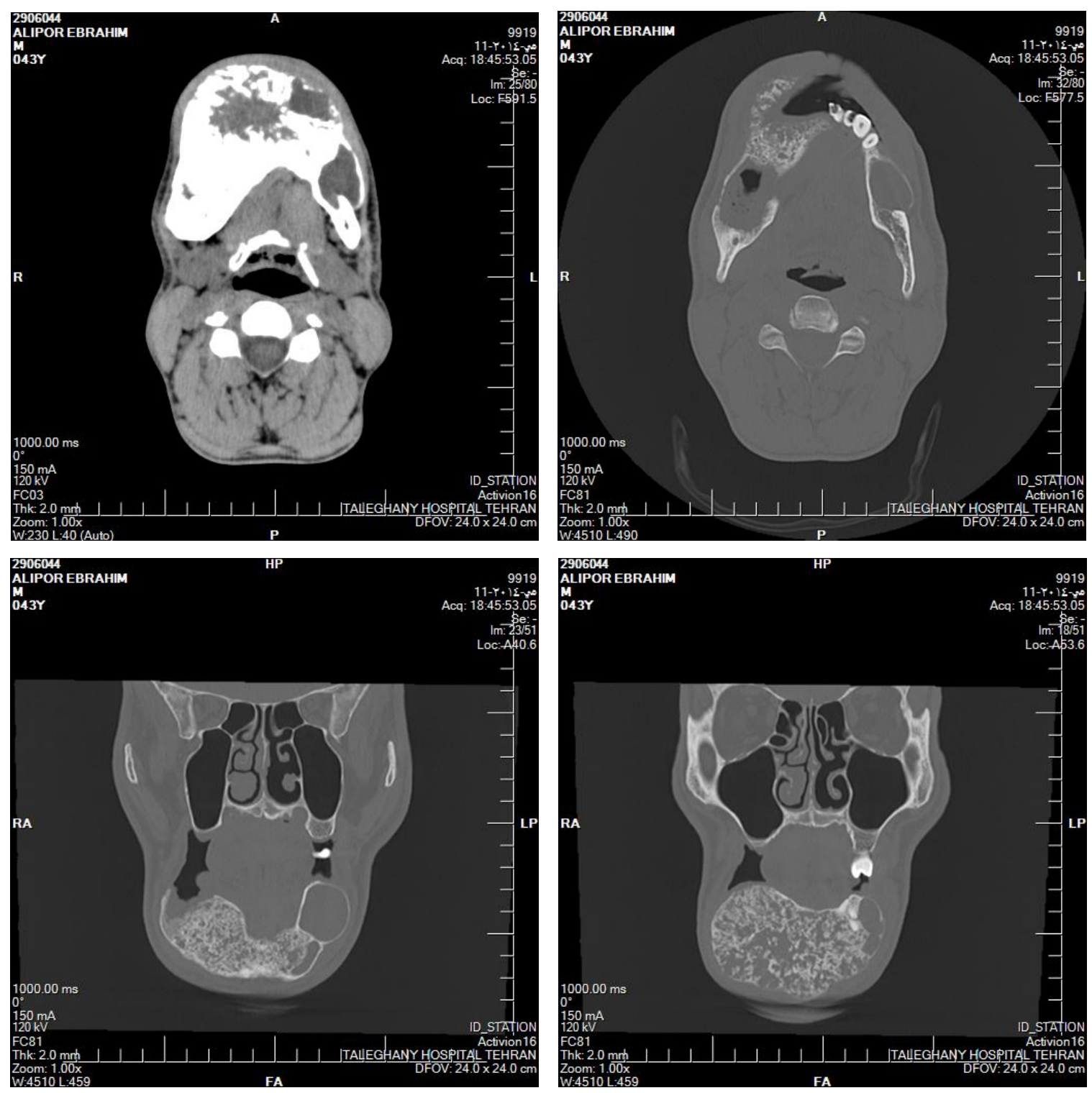

Figure 2. CT scan of the mandible (coronal and axial views).

The duration of symptoms until initial diagnosis has been reported to be 13 months in average [8]. In our case, the patient had swelling in the mandible (the most prevalent site of the tumor) with edformity and gradual spontaneous loss of the lower central teeth from about 12 months ago.

"Hybrid" ameloblastoma is a mixed variant of typical and desmoplastictype of the tumor". It is a rare type of the tumor (1\% - 4\%) [3]-[5] and was first described in detail by Waldron and El-Mofty in 1987 [10]. Takata et al. (1999) reviewed 8 cases of this type. They mentioned the tumors to be composed of two distinct sections (radiographically and histologically) [3]. One showed honeycomb soapbubble appearance with histologic features of desmoplastic ameloblastoma along with cystic features consistent with histologic characteristics of conventional type of the tumor (like our case) whereas others had uniform shape [11]. The honeycomb appearance of the desmoplastic portion of the tumor, seems to be caused by infiltrative behavior of the tumor. This may also explain the ill-defined border of the desmoplastic area of the tumor [3].

The prognosis of "hybrid" lesions are not yet clearly established. In our patient, local resection with safe margin of 15 millimeter was performed. There has been no recurrence in imaging after 6 months. Reichart and Philipsen reviewed 3677 patients with ameloblastoma. The rate of recurrence was $20.6 \%$ overally (14\% for mixed type) and the average time interval of 7 years was reported for all types of ameloblastoma (median of 5 years). 
The possibility and time of recurrence based on the method of therapy is also studied. Data has shown the possibility of recurrence to be $17 \%$ in radical therapy (the same as our patient) in comparison to conservative therapy with the recurrence risk of 34\% (with significant P-value) [8].

Different studies have discussed the pathogenesis of ameloblastoma. Theories have been proposed such as irritational factors, inflammation, nutritional deficiencies and viral infections. But none of them is completely proved. Human Papilloma Virus (HPV) antigen may have role in cell proliferation in tumor [12]. Monoclonality has been demonstrated in ameloblastoma. This finding gives strength to the idea that an initial mutation/molecular alteration is the first event in the development of the tumor. Different molecular alterations have been shown. Epidermal Growth Factor is more expressed in ameloblastoma cells [13]. The peripheral basal cell layer of ameloblastoma is shown to express anti-apoptotic proteins (such as $\mathrm{Bcl}-2$ ) together with cell proliferation markers (such as Ki67/MIB1) and therefore may be responsible for the progression of the tumor [14]. Matrix Metaloproteinases are enzymes that degrade extra-cellular matrix, are shown to be possible factors for invasiveness of the tumor [15].

\section{Conclusions}

Radiographic features reflect the histopathologic nature of tumors. As shown in this case, the mandible showed two distinct imaging patterns in the body and rami: the simple cystic appearance of the rami and the coarse soap bubble appearance of the body consistent with the conventional and desmoplastic types of Ameloblastoma respectively. The hybrid nature of the tumor should have been suggested by preoperative CT images with treatment implications since the unicystic variant of the tumor could be managed by bone curettage but the rest should be treated by resection with safe margin.

Radiographically, ameloblastoma can have a similar face to fibro-osseous lesions. The feature that can differentiate these two is the border of the tumor. Ameloblastoma tumors often have poorly defined borders, whereas fibro-osseous lesions usually have well-defined margins. Computed tomography can define the border and internal structure of the lesion and is particularly helpful in determining invasion to the near structures [16].

It is a universally practiced rule to biopsy a tumor from the site most representative of the lesion. In our case with two patterns of involvement, biopsies should have been taken from the mandibular body (as was performed) and the rami which could have suggested the hybrid variant prior to surgery. This is another example of how close the radiologists and clinicians should work together to plan the proper management and surgical approach, and also the importance of preoperative imaging evaluation. It also shows the radio-pathologic correlation of tumors and their behavior elegantly.

There was no apparent recurrence after 6 months in our patient. But data show that recurrence time is 7 years in average. [8] We should follow the patient to find the possible recurrence. The exact time of follow up is not clearly defined in the literature, thus more studies are needed in this area.

\section{Acknowledgements}

Our sincere thanks to colleagues in radiology department in Taleghani hospital who helped us in providing the images.

\section{Conflict of Interest}

The authors declare no potential conflicts of interest.

\section{Declaration}

All procedures followed were in accordance with the ethical standards of the responsible committee on human experimentation (institutional and national) and with the Helsinki Declaration of 1975, as revised in 2008. Informed consent was obtained from the patient for being included in the study.

\section{References}

[1] Torres-Lagares, D., Infante-Cossío, P., Hernández-Guisado, J.M. and Gutiérrez-Pérez, J.L. (2005) Mandibular Ameloblastoma. A Review of the Literature and Presentation of Six Cases. Medicina Oral Patologia Oral y Cirugia Bucal, 10, 231-238. 
[2] Taghavi, N., Rajabi, M., Mehrdad, L. and Sajjadi, S. (2013) A 10-Year Retrospective Study on Odontogenic Tumors in Iran. Indian Journal of Dental Research, 24, 220-224. http://dx.doi.org/10.4103/0970-9290.116688

[3] Takata, T., Miyauchi, M., et al. (1999) So-Called “Hybrid” Lesion of Desmoplastic and Conventional Ameloblastoma: Report of a Case and Review of the Literature. Pathology International, 49, 1014-1018. http://dx.doi.org/10.1046/j.1440-1827.1999.00972.x

[4] Lawal, A.O., Adisa, A.O., Olusanya, A.A. and Adeyemi, B.F. (2011) "Hybrid” Ameloblastoma: A Report of Two Cases. African Journal of Medicine \& Medical Sciences, 40, 413-415.

[5] Fulco, G.M., Nonaka, C.F., Souza, L.B., Miguel, M.C. and Pinto, L.P. (2010) Solid Ameloblastomas-Retrospective Clinical and Histopathologic Study of 54 Cases. Brazilian Journal of Otorhinolaryngology, 76, 172-177. http://dx.doi.org/10.1590/S1808-86942010000200005

[6] Bhandarwar, A.H., Bakhshi, G.D., Borisa, A.D., Wagh, A., Kapoor, R. and Kori, C.G. (2012) Anterior Mandibular Ameloblastoma. Clinical Practice, 14, e30. http://dx.doi.org/10.4081/cp.2012.e30

[7] Gupta, A. and Jindal, C. (2011) Hybrid Ameloblastoma: Report of a Rare Case and Review of Literature. International Journal of Oral and Maxillofacial Pathology, 2, 68-72.

[8] Reichart, P.A., Philipsen, H.P. and Sonner, S. (1995) Ameloblastoma: Biological Profile of 3677 Cases. European Journal of Cancer Part B: Oral Oncology, 31B, 86-99. http://dx.doi.org/10.1016/0964-1955(94)00037-5

[9] Tae Won Park, D.D.S. (1986) The Incidence and Classification of Ameloblastoma in Korea. Oral Radiology, 2, 19-24. http://dx.doi.org/10.1007/BF02347740

[10] Waldron, C.A. and El-Mofty, S.K. (1987) A Histopathologic Study of 116 Ameloblastomas with Special Reference to the Desmoplastic Variant. Oral Surgery, Oral Medicine, Oral Pathology, Oral Radiology, 63, 441-451. http://dx.doi.org/10.1016/0030-4220(87)90257-X

[11] dos Santos, J.N., De Souza, V.F., et al. (2006) "Hybrid” Lesion of Desmoplastic and Conventional Ameloblastoma: Immunohistochemical Aspects. Brazilian Journal of Otorhinolaryngology, 72, 709-713.

[12] Michael, A. and Kahn, D.D.S. (1989) Ameloblastoma in Young Persons: A Clinicopathologic Analysis and Etiologic Investigation. Oral Surgery, Oral Medicine, Oral Pathology, Oral Radiology, 67, 706-715. http://dx.doi.org/10.1016/0030-4220(89)90013-3

[13] Vered, M., Shohat, I. and Buchner, A. (2003) Epidermal Growth Factor Receptor Expression in Ameloblastoma. Oral Oncology, 39, 138-143. http://dx.doi.org/10.1016/S1368-8375(02)00034-9

[14] Gomes, C.C., Duarte, A.P., Diniz, M.G. and Gomez, R.S. (2010) Current Concepts of Ameloblastoma Pathogenesis. Journal of Oral Pathology \& Medicine, 39, 585-591. http://dx.doi.org/10.1111/j.1600-0714.2010.00908.x

[15] Jhamb, T. and Kramer, J.M. (2014) Molecular Concepts in the Pathogenesis of Ameloblastoma: Implications for Therapeutics. Experimental and Molecular Pathology, 97, 345-353. http://dx.doi.org/10.1016/j.yexmp.2014.09.001

[16] Rastogi, R., Jain, H., Mohan, C., Rastogi, V. and Rastogi, K. (2010) Hybrid Ameloblastoma—An Unusual Combination of Desmoplastic and Conventional Ameloblastoma. JIMSA, 23, 237-238. 